\title{
Can Subphotospheric Magnetic Reconnection Change the Elemental Composition in the
} Solar Corona?

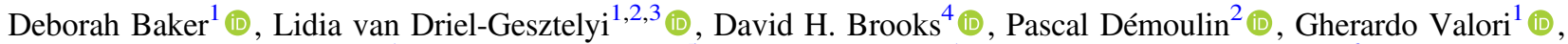 \\ David M. Long ${ }^{1}$ (D), J. Martin Laming ${ }^{5}$ (D), Andy S. H. To ${ }^{1}$ (i), and Alexander W. James ${ }^{6}$ (i) \\ ${ }^{1}$ University College London, Mullard Space Science Laboratory, Holmbury St. Mary, Dorking, Surrey, RH5 6NT, UK \\ ${ }^{2}$ LESIA, Observatoire de Paris, Université PSL, CNRS, Sorbonne Université, Univ. Paris Diderot, Sorbonne Paris Cité, 5 place Jules Janssen, \\ F-92195 Meudon, France \\ ${ }^{3}$ Konkoly Observatory, Research Centre for Astronomy and Earth Sciences, Hungarian Academy of Sciences, Konkoly Thege út 15-17., H-1121, Budapest, Hungary \\ ${ }_{5}^{4}$ College of Science, George Mason University, 4400 University Drive, Fairfax, VA 22030, USA \\ ${ }^{5}$ Space Science Division, Naval Research Laboratory, Code 7684, Washington, DC 20375, USA \\ ${ }^{6}$ European Space Astronomy Centre, Urb. Villafranca del Castillo, E-28692 Villanueva de la Cañada, Madrid, Spain \\ Received 2019 December 19; revised 2020 February 24; accepted 2020 March 3; published 2020 May 1
}

\begin{abstract}
Within the coronae of stars, abundances of those elements with low first ionization potential (FIP) often differ from their photospheric values. The coronae of the Sun and solar-type stars mostly show enhancements of low-FIP elements (the FIP effect) while more active stars such as M dwarfs have coronae generally characterized by the inverse-FIP effect (I-FIP). Here we observe patches of I-FIP effect solar plasma in AR 12673, a highly complex $\beta \gamma \delta$ active region. We argue that the umbrae of coalescing sunspots, and more specifically strong light bridges within the umbrae, are preferential locations for observing I-FIP effect plasma. Furthermore, the magnetic complexity of the active region and major episodes of fast flux emergence also lead to repetitive and intense flares. The induced evaporation of the chromospheric plasma in flare ribbons crossing umbrae enables the observation of four localized patches of I-FIP effect plasma in the corona of AR 12673. These observations can be interpreted in the context of the ponderomotive force fractionation model which predicts that plasma with I-FIP effect composition is created by the refraction of waves coming from below the chromosphere. We propose that the waves generating the I-FIP effect plasma in solar active regions are generated by subphotospheric reconnection of coalescing flux systems. Although we only glimpse signatures of I-FIP effect fractionation produced by this interaction in patches on the Sun, on highly active M stars it may be the dominant process.
\end{abstract}

Unified Astronomy Thesaurus concepts: The Sun (1693); Solar abundances (1474); Sunspots (1653); Solar flares (1496); Solar magnetic fields (1503)

Supporting material: animation

\section{Introduction}

Recent spectroscopic observations of the Sun have shown how the elemental composition of the solar atmosphere varies in space and time. The variability of composition appears to be intrinsically linked to the distribution and evolution of the Sun's magnetic field on all scales (e.g., Brooks et al. 2017; Baker et al. 2018). More generally, the overall composition of a star's corona depends on the first ionization potential (FIP) of the main elements comprising the corona. In solar-type stars, low-FIP elements are observed to be overabundant relative to their photospheric abundances, while high-FIP elements maintain their photospheric abundances (the FIP effect; e.g., Laming et al. 1995; Wood \& Linsky 2010; Laming 2015; Testa et al. 2015; Brooks et al. 2017). In cooler, more active stars such as M dwarfs, low-FIP/high-FIP elements are under-/ overabundant in the corona compared to the photosphere (the inverse FIP or I-FIP effect; Laming 2015; Brooks 2018). Wood \& Linsky (2010) and Wood et al. (2018) first established an almost linear relationship between stellar composition and spectral type $\mathrm{F}$ to $\mathrm{M}$ in the $\mathrm{X}$-ray spectra of moderately active stars (X-ray luminosity $<10^{29} \mathrm{erg} \mathrm{s}^{-1}$ ). In addition, a dependence has been found of coronal composition on stellar magnetic activity, with high-activity stars having I-FIP effect coronae and low-activity stars like our Sun having FIP-effect-dominated coronae (Audard et al. 2003; García-Alvarez et al. 2009; Testa et al. 2015).
One plasma fractionation model that is able to account for the FIP and I-FIP effects observed in stars is the ponderomotive force model (Laming 2015). The model invokes the ponderomotive force exerted by Alfvén waves giving rise to ionneutral separation in the chromosphere of the Sun and other stars. Fractionation takes place in the chromosphere where the temperature and density gradients are high and temperatures are such that low-FIP elements are mainly ionized and high-FIP elements remain neutral. The direction of the ponderomotive force determines whether low-FIP elements become enhanced or depleted in the corona. Downward propagating Alfvén waves generated by magnetic reconnection in the corona are mostly reflected back into the corona at the high density gradient in the chromosphere, creating an upward-directed ponderomotive force acting on the ions (Laming 2017). When the plasma is observed in the corona, the low-FIP elements are overabundant relative to the photosphere. The I-FIP effect is created when upward-traveling waves undergoing reflection or refraction back downwards produce a downward-directed ponderomotive force acting on the ions in the chromosphere. Such upward-propagating waves can be generated, for instance, by magnetoacoustic waves originating from below the chromosphere that are mode converted to fast-mode waves at the plasma $\beta=1$ boundary. As a consequence of the downward ponderomotive force, the plasma is then depleted of low-FIP elements (Laming 2015; Brooks 2018). In this article, we 
present observations that suggest a more specific origin for the waves producing the I-FIP effect, namely a subphotospheric one.

Though the Sun's corona is dominated by the FIP effect (Laming et al. 1995; Brooks et al. 2015, 2017), recent spectral scans taken by the EUV Imaging Spectrometer (EIS) (Culhane et al. 2007) on board the Hinode spacecraft (Kosugi et al. 2007) have shown highly localized regions of I-FIP effect plasma near sunspots in flare spectra (Doschek et al. 2015; Doschek \& Warren 2016, 2017). Doschek \& Warren (2017) proposed that the FIP effect "shuts down" near sunspots where p-modes are suppressed, leading to areas of photospheric, weak I-FIP, or I-FIP effect plasma, while the rest of the active region has coronal composition.

At low spatial resolution, solar coronal abundances in flares have been previously analyzed and shown to be very different from quiescent conditions (e.g., Sylwester et al. 1984; Feldman \& Widing 1990; Warren 2014), having a tendency to be close to photospheric values. Composition studies of stellar flares provide strong indications of a similar effect, i.e., that the elemental abundances tend to approach photospheric values both on FIP-effect and I-FIP-effect dominated stars (e.g., Nordon \& Behar 2008; Laming \& Hwang 2009). Recently, Katsuda et al. (2020) reported the I-FIP effect in four large X-class flares (X17.0, X5.4, X6.2 from AR 10808 and X9.0 from AR 10930) derived from Earth albedo X-ray spectra from the imaging spectrometer on board the Suzaku astronomical satellite.

Baker et al. (2019) analyzed in detail Hinode/EIS observations of plasma composition during the decay phase of an M-class flare in AR 11429. Patches of I-FIP effect plasma appeared in a highly sheared emerging flux region above sunspot umbrae 10 minutes after the flare peak and disappeared 40 minutes later. The authors proposed that subchromospheric reconnection of highly sheared coalescing strands of the same polarity magnetic field played a key role in the creation of I-FIP effect plasma within the highly complex magnetic field of the active region. During episodes of strong emergence, flux approached and interacted with preexisting magnetic field, forcing the coalescence of the smaller flux fragments into growing, coherent umbrae surrounded by common penumbrae in two of the sunspots. The convergence of magnetic field in the location of the coalescing umbrae is highly suggestive of the presence of subchromospheric magnetic reconnection. Reconnection that occurs below the region of plasma fractionation in the chromosphere generates a fast-mode wave flux in the direction required by the ponderomotive force fractionation model to produce I-FIP effect plasma (Laming 2015). The fractionated plasma is then evaporated into the corona by flaring and observed by Hinode/EIS when flare ribbons cross the coalescing umbra.

In this paper, magnetic field and continuum observations of the most unusual and complex magnetic field of AR 12673 are combined with Hinode/EIS scans to investigate the presence of I-FIP effect plasma. We confirm the findings of Baker et al. (2019) and provide evidence in support of the generation of I-FIP effect plasma by subphotospheric reconnection in coalescing sunspot umbrae with strong light bridges (LBs). The paper is organized as follows: Section 2 describes the magnetic field evolution, Section 3 details the Hinode/EIS observations, Section 4 provides our analysis and interpretation of the observations of anomalous plasma composition in the context of the ponderomotive force fractionation model, and in Section 5 we present our conclusions.

\section{Magnetic Field Evolution of AR 12673}

AR 12673 was visible on the Sun from 2017 August 28 to September 10. Its magnetic field evolved into one of the most complex structures observed during solar cycle 24 (L. van DrielGesztelyi et al. 2020, in preparation). The sunspot group's Mount Wilson magnetic classification was $\beta \gamma \delta$, the total unsigned flux exceeded $5 \times 10^{22} \mathrm{Mx}$ and the projected whole spot area peaked on September 6 at $2100 \mathrm{MSH}$ (millionths of a solar hemisphere; from the Debrecen Photoheliographic Database: http://fenyi. solarobs.csfk.mta.hu/DPD/2017/index.html). Overall activity included 4 X-, 27 M-, and 55 C-class flares, making AR 12673 the most flare productive of solar cycle 24. Several significant coronal mass ejections (CMEs) were launched during its disk passage (Redmon et al. 2018). Furthermore, this "monster" active region exhibited highly unusual characteristics and extreme behavior including the fastest flux emergence ever recorded (Sun \& Norton 2017), the strongest transverse, (Wang et al. 2018) and coronal magnetic fields (Anfinogentov et al. 2019).

Figure 1 shows selected cotemporal radial magnetic field and continuum images from the Helioseismic and Magnetic Imager (HMI; Scherrer et al. 2012) on board the Solar Dynamics Observatory (SDO; Scherrer et al. 2012). The data are from the Spaceweather HMI Active Region Patch (SHARP; Bobra et al. 2014) pipeline. Figure 1 includes an animation of these data for 2017 September 1-7. A full account of the structure and evolution of the magnetic field leading to the first direct evidence of subphotospheric reconnection is provided in L. van Driel-Gesztelyi et al. (2020, in preparation). The main features are summarized here using the same notation.

AR 12673 was formed from the emergence and subsequent interaction of three flux systems labeled in Figure 1: a longlived, stable positive spot $\mathrm{P}_{0}$; an arcade system formed of $\mathrm{n}_{1}-\mathrm{p}_{1}$ and $\mathrm{n}_{2}-\mathrm{p}_{2}$; and the main flux system composed of $\mathrm{N}_{3}-\mathrm{P}_{3}$ and $\mathrm{N}_{4}-\mathrm{P}_{4}$ (the arcade/main flux systems are labeled in gray/black in Figure 1). Early on September 2, bipole $n_{1}-p_{1}$ emerged to the southeast of preexisting $P_{0}$. Bipole $n_{2}-p_{2}$ followed one day later and emerged to the northeast of $\mathrm{P}_{0}$. Very quickly the two bipoles formed a coherent arcade system with a C-shaped magnetic polarity inversion line (PIL), as the fast moving emerging flux wrapped around the well-anchored $\mathrm{P}_{0}$. On September 3, the main field comprising bipoles $\mathrm{N}_{3}-\mathrm{P}_{3}$ and $\mathrm{N}_{4}-\mathrm{P}_{4}$ emerged in a dominantly $\mathrm{N}-\mathrm{S}$ direction underneath the E-W oriented arcade system represented by $n_{1}-p_{1}$ and $n_{2}-p_{2}$. At the location where the main system emerged into the arcade system lying above it, an M-shaped flux tube was present. It was formed by a concave-up U-loop (van Driel-Gesztelyi et al. 2000) between $\mathrm{N}_{4}$ and $\mathrm{P}_{3}$ and two lobes of concave-down $\Omega$ loops, $\mathrm{P}_{3}-\mathrm{N}_{3}$ in the north and $\mathrm{P}_{4}-\mathrm{N}_{4}$ in the south (Fan et al. 2003; Toriumi et al. 2014).

Key aspects of the active region's magnetic field evolution occurred in the time period of September 4-6 leading up to the times of the X2.2 and X9.3 flares. First, during the ongoing emergence of the constituent bipoles of the three flux systems, strands of the same polarity magnetic field coalesced to form coherent sunspot umbrae in the active region core to the east of $\mathrm{P}_{0}\left(\right.$ at $\mathrm{p}_{2}$ and $\left.\mathrm{N}_{4}\right)$ and at its periphery to the north and southwest 
04 Sept 00:10

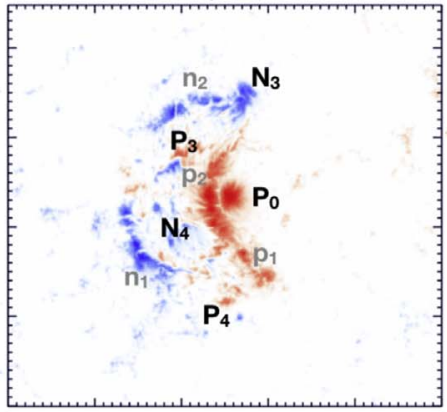

06_Sept_02:34

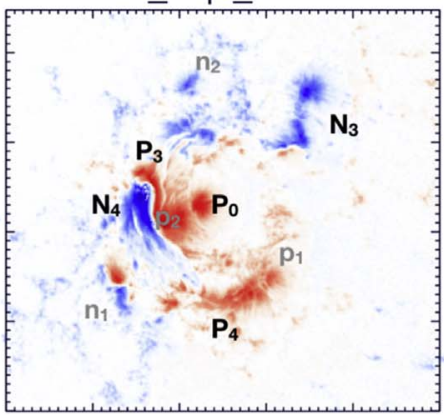

06_Sept_19:34

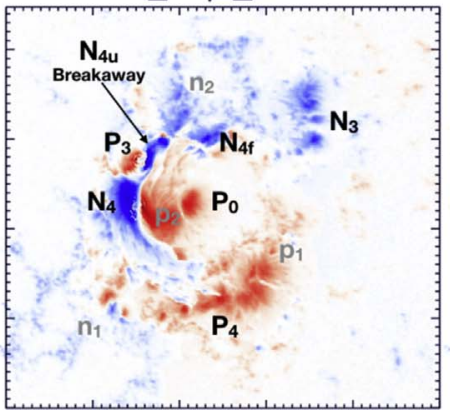

05_Sept 08:34
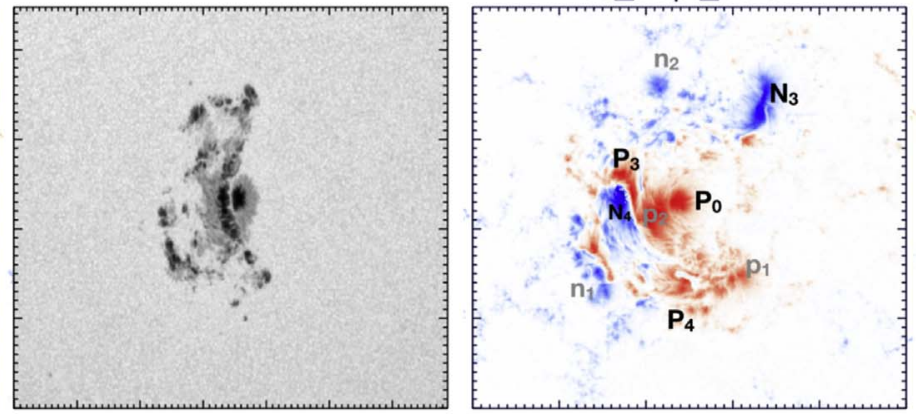

06_Sept_16:46
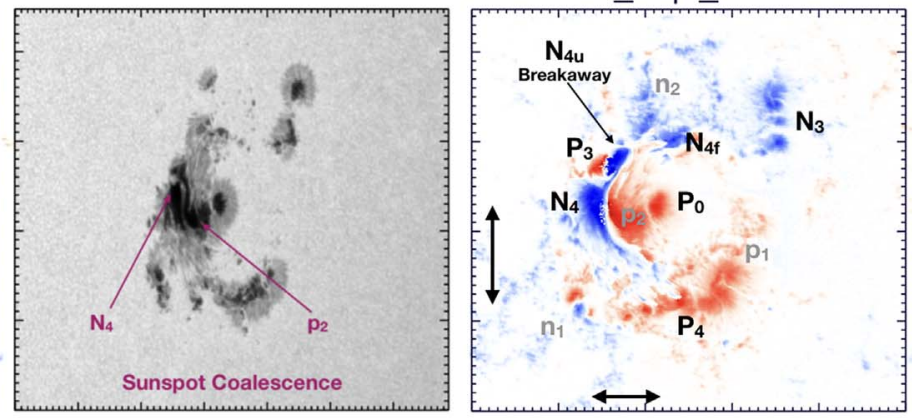

07 Sept 08:34

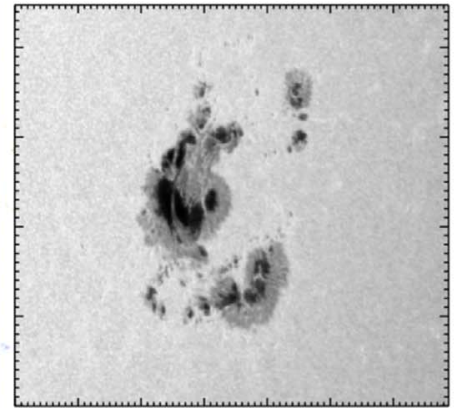

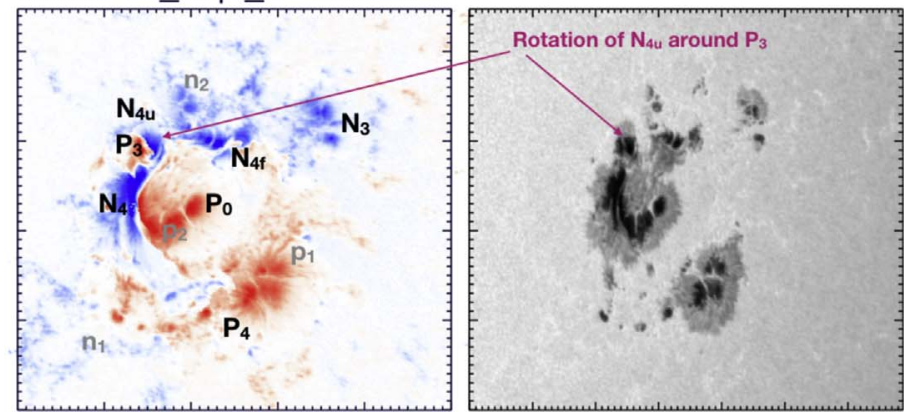

Figure 1. SDO/HMI SHARP radial magnetic field, $B_{r}$, and $6173 \AA$ A continuum images (Bobra et al. 2014) during the evolution in AR 12673 . The middle and bottom panels correspond to the times of when Hinode/EIS observed I-FIP effect plasma (Figures 3-5). Major components of the flux systems are labeled in each image: arcade field $\mathrm{n}_{1}-\mathrm{p}_{1}$ and $\mathrm{n}_{2}-\mathrm{p}_{2}$ (labels in gray); main field $\mathrm{N}_{3}-\mathrm{P}_{3}$ and $\mathrm{N}_{4}-\mathrm{P}_{4}$ (labels in black), and preexisting, well-anchored spot $\mathrm{P}_{0}$. The U-loop is formed in between $\mathrm{N}_{4}-\mathrm{P}_{3}$ as well as $\mathrm{N}_{4 u}-\mathrm{P}_{3}$ (bottom panels). Near the end of the emergence phase, polarities $\mathrm{N}_{4 u}$ and $\mathrm{N}_{4 f}$ detached from the north part of $\mathrm{N}_{4}$. Note that $\mathrm{n}$ and $\mathrm{N}$ notations are used for negative, $\mathrm{p}$ and $\mathrm{P}$ notations for positive polarity umbrae. Red/blue corresponds to positive/negative $B_{r}$ in the magnetograms. For ease of comparison between figures, black arrows are used to show the distances from the center of the major polarities. At 16:46 UT on September 6, the vertical arrow is $\sim 50^{\prime \prime}$ in length between the centers of $\mathrm{P}_{0}$ and $\mathrm{P}_{4}$. The horizontal arrow is $\sim 30^{\prime \prime}$ in length from the centers of $\mathrm{P}_{0}$ and $\mathrm{N}_{4}$. The center of $\mathrm{P}_{0}$ is $X=590^{\prime \prime}, Y=-242^{\prime \prime}$. This convention is repeated for all figures with maps. The animated images are not annotated and span from 2017 September 1-7 at approximately 12 minutes per frame.

(An animation of this figure is available.)

at $\mathrm{n}_{2}$ and $\mathrm{p}_{1}$, respectively. These were also the locations where significant LBs formed between merging umbral flux strands. Coalescence was driven by especially strong and fast episodes of flux emergence on September 4-6 (see the animated version of Figure 1). Second, early on September 6, the north-western part of $\mathrm{N}_{4}$ detached and began moving northward. This detached polarity penetrated the "wall" of opposite polarity field along $\mathrm{P}_{3}-\mathrm{p}_{2}$ (Figure 1, middle panels), then it broke in two pieces. One piece, $\mathrm{N}_{4 u}$, mostly rotated counterclockwise around $\mathrm{P}_{3}$ indicating that it was still bound by the U-loop anchorage (Figure 1, bottom panels). The other piece, $\mathrm{N}_{4}$, moved rapidly northward, then westward in the direction of $\mathrm{N}_{3}$. On September 7 , the main flux system-at least temporarily-ceased its emergence into the $\mathrm{E}-\mathrm{W}$ arcade flux system, thereby removing the major driver of the unusual and extreme magnetic field evolution in AR 12673.

\section{Observations of AR 12673}

In this analysis, we focus on the three day period encompassing the largest flare of solar cycle 24, the X9.3 flare that peaked at $\sim 11: 53$ UT on September 6 . Figure 2 shows the GOES 1-8 $\AA$ soft X-ray light curve from 00:00 UT on September 5-00:00 UT on September 8 when Hinode/EIS was observing AR 12673 employing a multitude of studies and scanning modes. However, not all studies contain suitable emission lines from high- and low-FIP elements for measuring plasma composition. The basic details of the studies used here are provided in Table 1. The times of the Hinode/EIS composition observations are indicated by the dashed red lines plotted with the GOES soft X-ray curve in Figure 2.

The studies listed in Table 1 contain the high-FIP Ar XIV $194.40 \AA(\mathrm{FIP}=15.76 \mathrm{eV})$ and low-FIP Ca XIV $193.87 \AA$ $(\mathrm{FIP}=6.11 \mathrm{eV})$ emission lines. The two ions have similar 


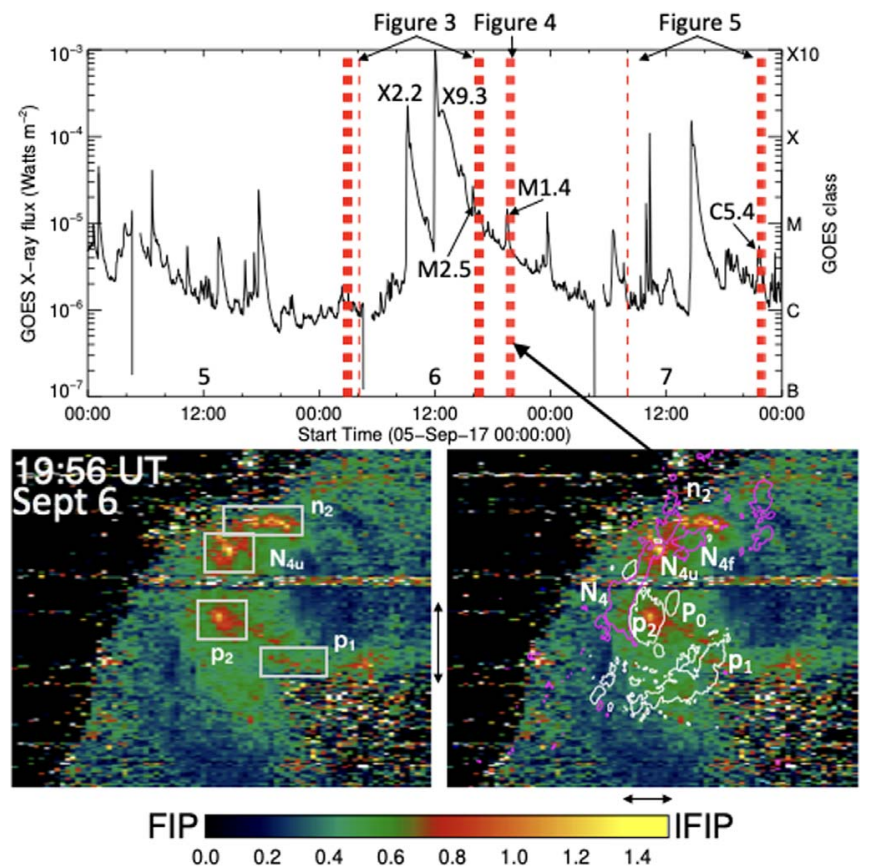

Figure 2. Top panel: GOES 1-8 $\AA$ soft X-ray light curve from 00:00 UT on 2017 September 5 to 00:00 UT on 2017 September 8. Red dashed lines indicate the times of Hinode/EIS rasters used in this study. The thicker lines represent a series of nearby observations. Bottom panels: Hinode/EIS Ar XIV/Ca XIV ratio map at 19:56 UT on September 6 overplotted with boxes defining the regions used in Table 2 and throughout the text (left) and SDO/HMI contours of $\pm 500 \mathrm{G}$ of white/magenta for positive/negative polarities (right). The color bar scale shows the FIP effect as blue/green, the photospheric composition as orange, and the I-FIP effect as yellow. $\left(\mathrm{P}_{0}\right.$ is located at $X=611^{\prime \prime}, Y=-241^{\prime \prime}$ at 19:56 UT.)

Table 1

Hinode/EIS Study Details

\begin{tabular}{|c|c|}
\hline Study Name & HIC2_SCAN_201 $\times 512$ \\
\hline $\begin{array}{l}\text { Composition } \\
\text { Field of view } \\
\text { Rastering } \\
\text { Exposure time }\end{array}$ & $\begin{array}{c}\text { Ca XIV 193.87 } \AA \text { and Ar XIV } 194.40 \AA \\
210^{\prime \prime} \times 512^{\prime \prime} \\
2^{\prime \prime} \text { slit, } 21 \text { positions, } 10^{\prime \prime} \text { coarse steps } \\
15 \mathrm{~s}\end{array}$ \\
\hline $\begin{array}{l}\text { Study name } \\
\text { Composition lines } \\
\text { Field of view } \\
\text { Rastering } \\
\text { Exposure time }\end{array}$ & $\begin{array}{c}\text { Atlas_60 } \\
\text { Full spectral atlas including } \\
\text { Ca XIV 193.87 } \AA \text { and Ar XIV 194.40 } \\
120^{\prime \prime} \times 160^{\prime \prime} \\
2 \text { " slit, } 60 \text { positions, 2" steps } \\
60 \mathrm{~s}\end{array}$ \\
\hline $\begin{array}{l}\text { Study name } \\
\text { Composition lines } \\
\text { Field of view } \\
\text { Rastering } \\
\text { Exposure time }\end{array}$ & $\begin{array}{c}\text { FlareResponse01 } \\
\text { Ca XIV 193.87 } \AA \text { and Ar XIV } 194.40 \AA \\
240^{\prime \prime} \times 304^{\prime \prime} \\
2^{\prime \prime} \text { slit, } 80 \text { positions, } 3^{\prime \prime} \text { coarse steps } \\
5 \mathrm{~s}\end{array}$ \\
\hline
\end{tabular}

contribution functions, therefore their intensity ratio is suitable to determine (I)FIP levels within the active region (Feldman et al. 2009). In line with Doschek et al. (2015), Doschek \& Warren (2016, 2017), and Baker et al. (2019), we use the following $\log _{10}$ abundance values relative to $\log _{10} \mathrm{H}=12$ : $\mathrm{Ca}=6.93$ (Feldman 1992) and 6.33 (Caffau et al. 2011) for the corona and photosphere, respectively; $\mathrm{Ar}=6.5$ (Lodders 2008) is the same for the corona and the photosphere. Calculations of the contribution functions using these abundances were performed
Table 2

Mean I-FIP Effect Ratio Values on September 6 in Regions Defined in Figure 2 with the Selection of Pixels having Ratio Values $\geqslant 1.3$

\begin{tabular}{lcccc}
\hline \hline Raster Times (UT) & $\mathrm{n}_{2}$ & $\mathrm{~N}_{4 u}$ & $\mathrm{p}_{2}$ & $\mathrm{p}$ \\
\hline $04: 11$ & $\ldots$ & 1.5 & $\ldots$ & $\ldots$ \\
$16: 19$ & 1.5 & 1.4 & $\ldots$ & $\ldots$ \\
$16: 25$ & $\ldots$ & 1.4 & $\ldots$ & $\ldots$ \\
$16: 31$ & $\ldots$ & 1.5 & $\ldots$ & $\ldots$ \\
$16: 37$ & $\ldots$ & 1.4 & $\ldots$ & $\ldots$ \\
$16: 43$ & $\ldots$ & 1.3 & $\ldots$ & 1.4 \\
$16: 49$ & $\ldots$ & 1.3 & $\ldots$ & 1.4 \\
$16: 55$ & $\ldots$ & 1.3 & $\ldots$ & 1.3 \\
$17: 01$ & 1.3 & 1.4 & $\ldots$ & $\ldots$ \\
$19: 29$ & 1.8 & $\ldots$ & $\ldots$ & 1.3 \\
$19: 38$ & 1.3 & $\ldots$ & 1.5 & 1.8 \\
$19: 47$ & 1.4 & $\ldots$ & 1.3 & 1.3 \\
$19: 56$ & 1.6 & 1.4 & 1.3 & 1.4 \\
$20: 05$ & 1.4 & 1.4 & $\ldots$ & 1.5 \\
$20: 14$ & 1.4 & 1.3 & $\ldots$ & 1.5 \\
\hline
\end{tabular}

with the software included in the CHIANTI Atomic Database, version 8.0 (Dere et al. 1997; Del Zanna et al. 2015). Based on the intensity ratio of the contribution functions of high-FIP Ar XIV and low-FIP Ca XIV, values $>1$ indicate the I-FIP effect, $=1$ is photospheric plasma, and $<1$ is FIP effect or typical solar coronal plasma. The estimated uncertainty of the ratio is \pm 0.28 assuming an intensity error of $20 \%$. Considering the uncertainties, in the text and figures we refer to ratio values $\geqslant 1.3$ as I-FIP effect plasma.

Though Ar XIV and Ca XIV have similar contribution functions, it is not immediately apparent what, if any, temperature effects flaring might have on the uncertainties of the I-FIP effect measurements, as large flares can have much higher densities $(N)$ at high temperatures $(T)$ (e.g., Watanabe et al. 2010; Graham et al. 2011; Simões et al. 2015) compared to nonflaring active regions (Doschek et al. 2007; Tripathi et al. 2008). The Ar XIV/Ca XIV ratio can vary by a factor $\sim 4$ in the $\log _{10} T=6.6-6.9$ range (with $T$ in $\mathrm{K}$, see e.g., Doschek \& Warren 2017; Baker et al. 2019).

We derived the mean temperatures and densities for all strong I-FIP effect regions corresponding to Table 2 where the appropriate diagnostic line pairs were included in the EIS studies (e.g., Ar XIV 187.9/194.4 $\AA$ and Fe XIII 203.8/ 202.0 ). For raster times from 19:29 to 20:14 UT, we were only able to obtain temperature measurements. $\log _{10} N$ were in the range 10.2-11.1 (with $N$ in $\mathrm{cm}^{-3}$ ) and the $\log _{10} T$ were 6.6-6.7. These compare to values in FIP effect regions of $\log _{10}$ $N=8.9-9.9$ and $\log _{10} T=6.2-6.4$, respectively.

Higher density in the I-FIP effect regions lowers the theoretical ratio, so high observed I-FIP effect values of $\mathrm{Ar}$ $\mathrm{XIV} / \mathrm{Ca}$ XIV become more extreme in the abundance differences that they imply. Furthermore, the temperatures fall within the range that is considered to be plausible for the formation temperatures of Ar XIV and Ca XIV (Doschek \& Warren 2017). It is very difficult to disentangle the density and temperature effects caused by the flaring but, the increase in density combined with the fact that the temperature does not exceed $\log _{10} T=6.7$ suggests that the density and temperature effects are unlikely to substantially affect the estimated uncertainty of \pm 0.28 . The results may be very different for measurements made during the peak phase of large flares, but 
this is not the case here as the rasters were timed during the decay phases of the flares on September 6.

Spectroscopic data were reduced using standard routines available in the Hinode/EIS section of Solar Software (SSW; Freeland \& Handy 1998). Three Gaussians were fit to the Ar XIV $194.40 \AA$ emission line to remove two unidentified lines in its blue wing. The Ca XIV $193.87 \AA$ line was also fit with three Gaussians to separate the line from nearby Fe X $193.72 \AA$, Ni XVI 194.05 ̊, and 194.10 ̊ lines (Brown et al. 2008; Doschek et al. 2015; Baker et al. 2019).

All Hinode/EIS maps and SDO/HMI line-of-sight magnetograms were coregistered in a two step process. First, the aia_prep.pro routine located in the SDO branch of SSW was used to align HMI and Atmospheric Imaging Assembly (AIA) data. The routine takes into account differences in the plate scales and roll angles between the two instruments. Second, either the Fe XII $195.12 \AA$ or $186.88 \AA$ EIS intensity maps were aligned by eye with the cotemporal AIA $193 \AA$ passband images. The EIS Fe XII emission lines and AIA $193 \AA$ broadband imager sample plasma at similar temperatures so that the same coronal structures are identifiable in each image, making alignment straightforward between Hinode/EIS and SDO. To help the reader to track different features in the figures, we have added arrows to indicate the central positions of major polarities and the distances between them. A vertical arrow shows the positions and distance of the $\mathrm{P}_{0}-\mathrm{P}_{4}$ centers $\left(\sim 50^{\prime \prime}\right)$ and a horizontal arrow shows the same for $\mathrm{P}_{0}-\mathrm{N}_{4}$ $\left(\sim 30^{\prime \prime}\right)$.

Figure 2, lower panel, shows a sample Ar XIV/Ca XIV ratio map with/without SDO/HMI contours of $\pm 500 \mathrm{G}$ (white/ magenta for positive/negative polarities) at 19:56 UT on September 6. Four regions of interest based on the magnetic flux systems described in Section 2 are labeled as follows: $\mathrm{n}_{2}$ in the spatially extended magnetic field to the north; $\mathrm{N}_{4 u}$ which detached northward from $\mathrm{N}_{4} ; \mathrm{p}_{2}$ located on the east side of the large positive sunspot $\mathrm{P}_{0}$; and $\mathrm{p}_{1}$ along the extended positive polarity to the south. Similar ratio maps with the corresponding Ar XIV $194.40 \AA$ and Ca XIV $193.87 \AA$ intensity maps are displayed in Figures 3 and 4.

Hinode/EIS observed AR 12673 at 02:35 UT on September 6 during a relatively quiet period (Figure 3, top panel). The core of the active region is predominantly composed of typical coronal plasma. FIP effect levels range from $\sim 0.3$ to 0.5 using the high-FIP Ar XIV/low-FIP Ca XIV ratio, which is equivalent to the conventional solar low-FIP/high-FIP ratio of 2.0-3.3. Localized regions at $\mathrm{p}_{2}$ and $\mathrm{N}_{4}$ show weak indications of I-FIP effect plasma. Both patches of I-FIP effect plasma evolve to photospheric composition within $\sim 25$ minutes (at 02:59 UT) and the patch associated with $\mathrm{N}_{4}$ returns to coronal plasma within $\sim 50$ minutes (at 03:23 UT) when Hinode/EIS composition observations ended.

EIS composition observations resumed at 16:13 UT during the extended decay phase of the X9.3 flare, just after the peak of an M2.5 flare at 15:56 UT (see the GOES soft X-ray light curve in Figure 2). At this time plasma of photospheric composition was present in the southern part of $\mathrm{n}_{2}$ and at $\mathrm{N}_{4 u}$. Thirty minutes later, the plasma at $\mathrm{N}_{4 u}$ had evolved to I-FIP effect plasma while weak I-FIP patches appeared at $\mathrm{p}_{2}$ (Figure 3, bottom panel). I-FIP effect plasma was observed at these locations until the Hinode/EIS observing sequence ended at 17:07 UT
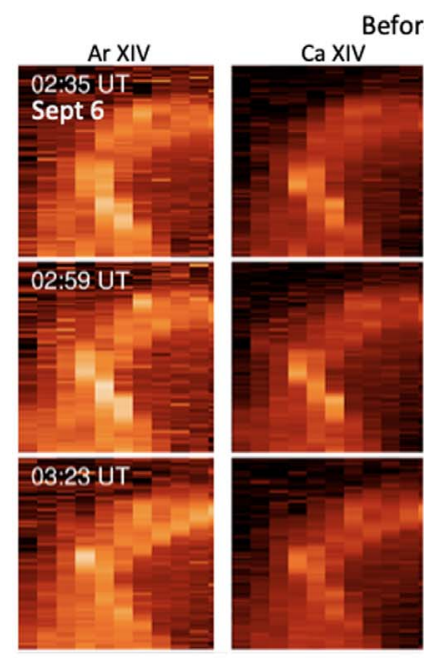

Before flare
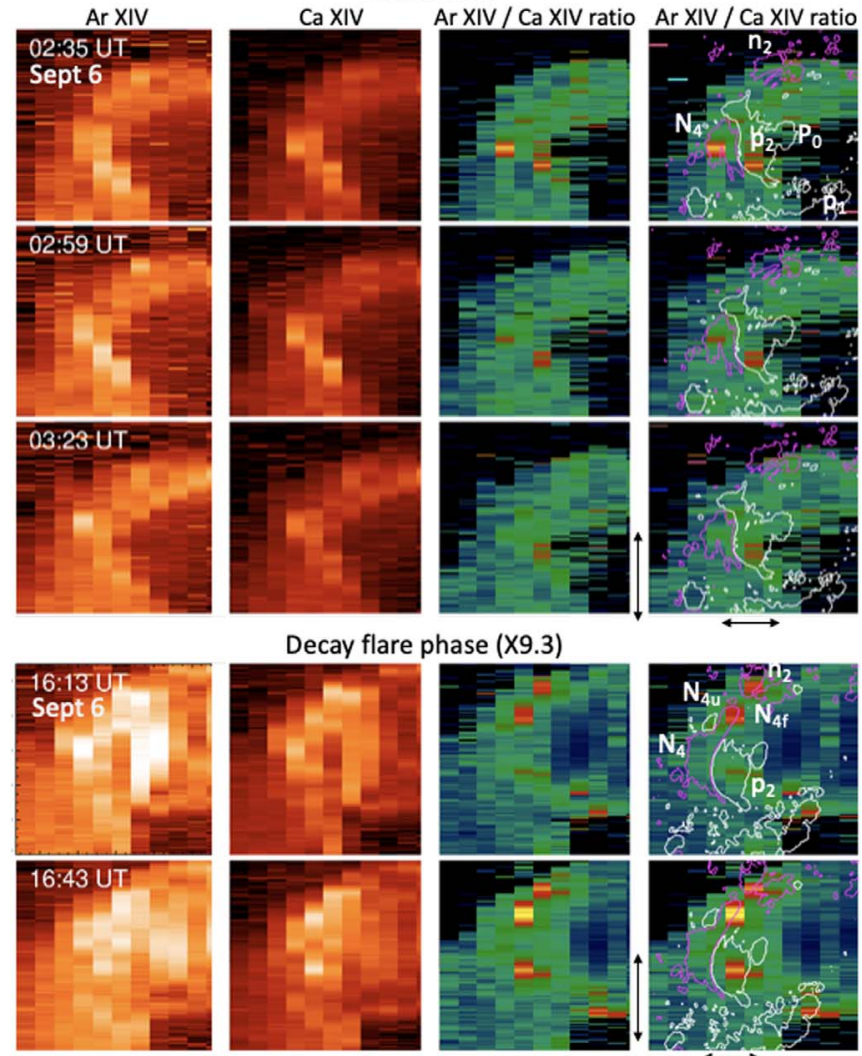

Decay flare phase (X9.3)
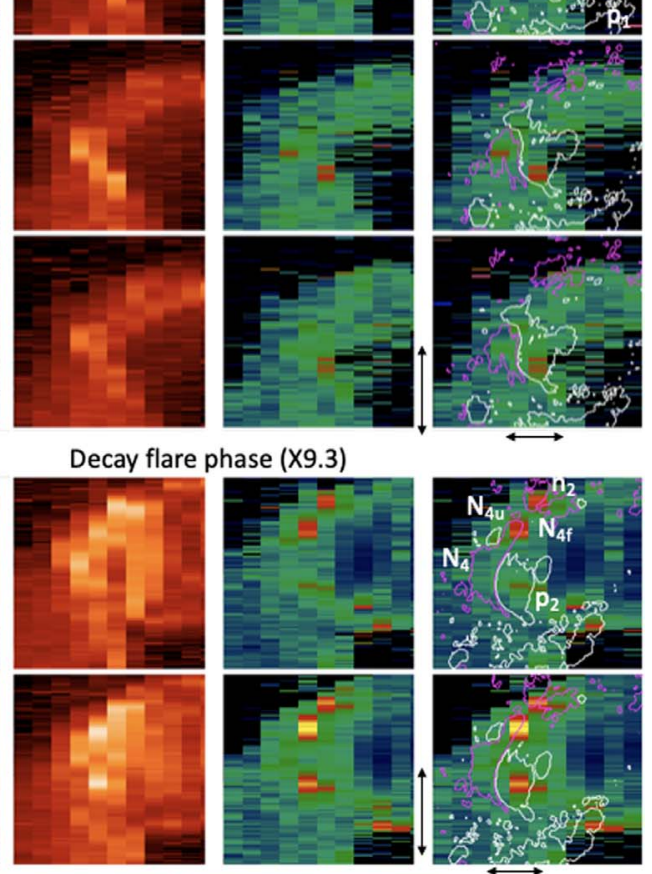

FIP

$\begin{array}{llllllll}0.0 & 0.2 & 0.4 & 0.6 & 0.8 & 1.0 & 1.2 & 1.4\end{array}$

Figure 3. Left to right: Hinode/EIS Ar XIV $194.40 \AA$ and Ca XIV $193.87 \AA$ intensity maps, Ar XIV/Ca XIV ratio maps without and with SDO/HMI contours of \pm 500 (white/magenta for positive/negative polarities) before (top section) and during the decay phase of the X9.3 flare (bottom section) on 2017 September 6 . The color bar scale shows the FIP effect as blue/green, the photospheric composition as orange, and the I-FIP effect as yellow. All Hinode/EIS maps are coaligned to SDO/HMI maps at the times shown. $\left(\mathrm{P}_{0}\right.$ is located at $X=492^{\prime \prime}, Y=-248^{\prime \prime}$ at 03:23 UT and $X=590^{\prime \prime}, Y=-242^{\prime \prime}$ at 16:43 UT.)

The data shown in Figure 4 were obtained when Hinode/EIS was operating in its autonomous observing mode. A flare response study was triggered by an M1.4 flare at 19:29 UT on September 6. The large field of view (FOV) is centered on the active region and extends to the flare loops, which is not the case in Figure 3 where the smaller FOV covers only the core of the active region (see the FOVs shown in white in Figure 4). All regions defined in Figure 2 have patches of I-FIP effect plasma from 19:29-20:14 UT except for $\mathrm{p}_{2}$ where the plasma showing weak indications of the I-FIP effect evolved to photospheric composition from 20:05 to 20:14 UT.

The mean I-FIP effect ratios for pixels with values $\geqslant 1.3$ for each of the regions defined in the lower left panel of Figure 2 are given for Hinode/EIS rasters on September 6 in Table 2. In general, I-FIP effect plasma persisted in the $\mathrm{n}_{2}$ and $\mathrm{p}_{1}$ regions and at $\mathrm{N}_{4 u}$, but less so within $\mathrm{p}_{2}$.

On September 7 at 08:01 UT, photospheric plasma was present within the flare loops associated with $\mathrm{p}_{2}$ and at both pieces of the well-separated breakaway negative polarity $\mathrm{N}_{4 u}$ and $\mathrm{N}_{4 f}$ in the top panel of Figure 5. This single Hinode/EIS observation was acquired during the decay phase of a C8.2 flare that peaked at 06:42 UT. The plasma within the $\mathrm{p}_{1}$ region 


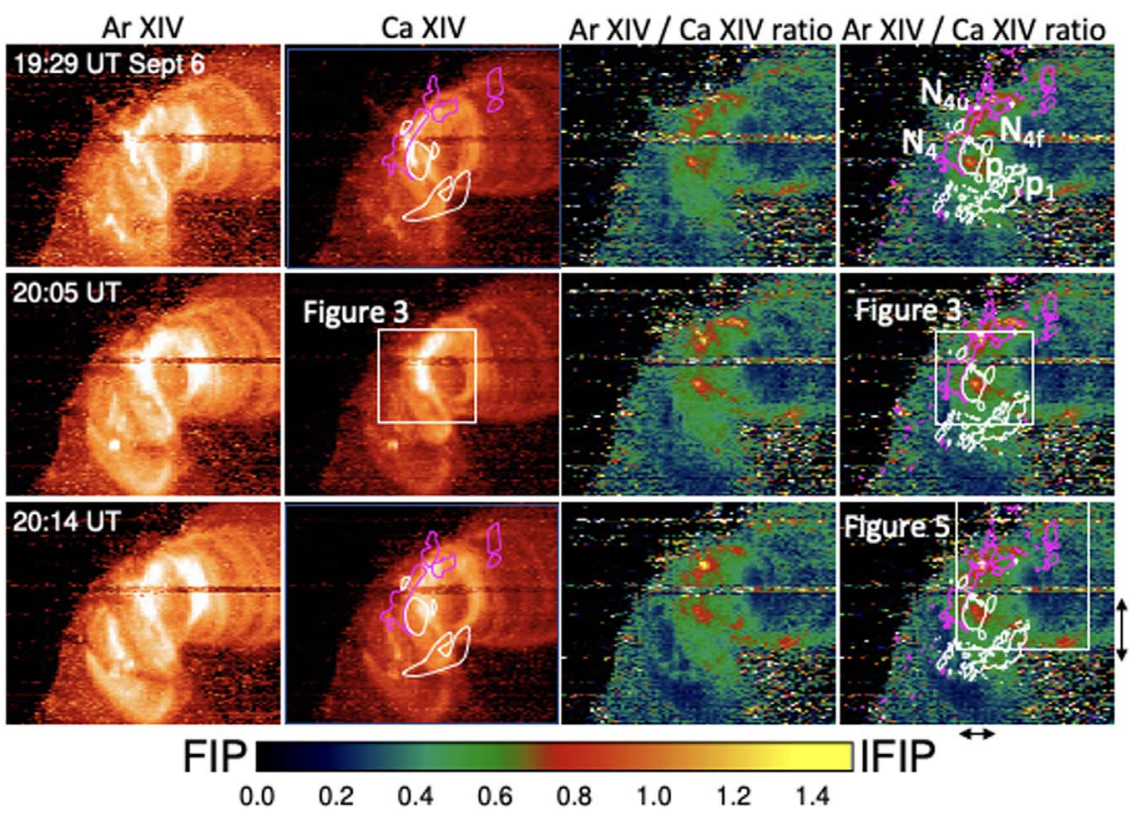

Figure 4. Left to right: Hinode/EIS Ar XIV $194.40 \AA$ and Ca XIV $193.87 \AA$ intensity maps, Ar XIV/Ca XIV ratio maps without and with SDO/HMI contours of \pm 500 (white/magenta for positive/negative polarities). Observations are from 19:29 UT to 20:14 UT on 2017 September 6. The color bar scale shows the FIP effect as blue/green, the photospheric composition as orange, and the I-FIP effect as yellow. All Hinode/EIS maps are coaligned to SDO/HMI maps at the times shown. $\left(\mathrm{P}_{0}\right.$ is located at $X=614^{\prime \prime}, Y=-241^{\prime \prime}$ at 20:14 UT.)

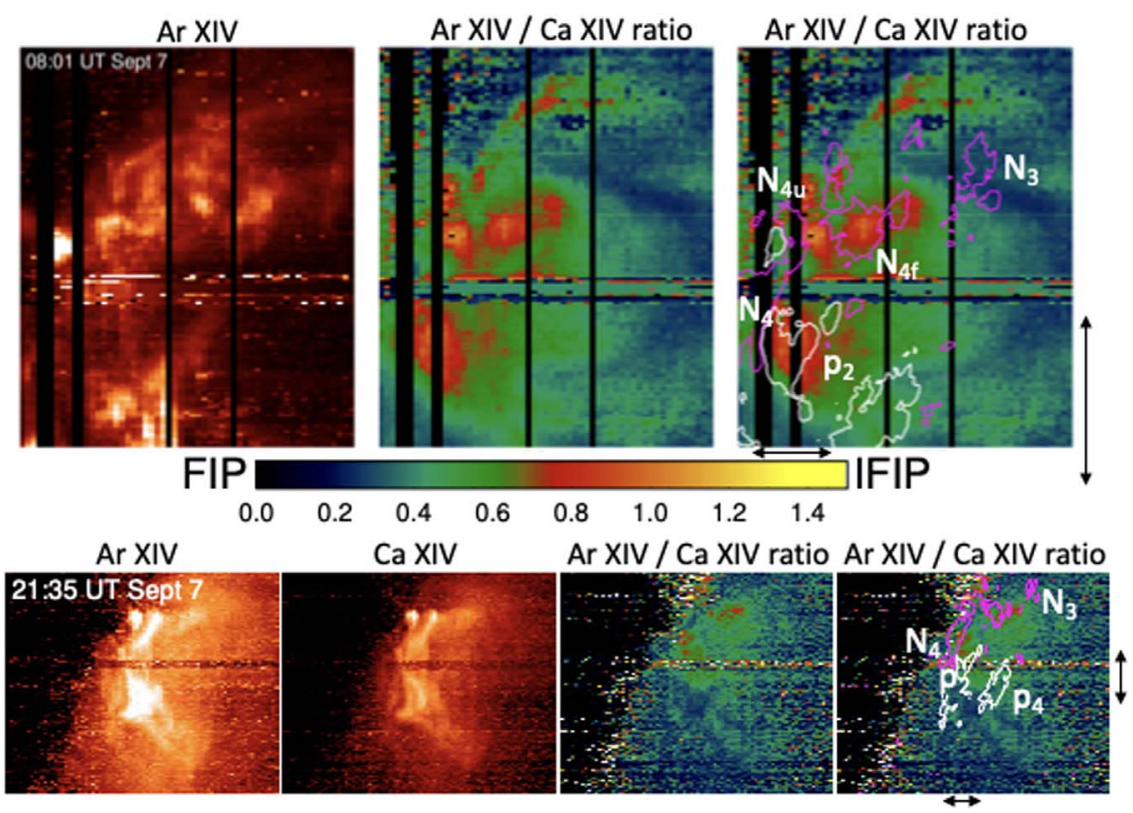

Figure 5. Top panel: Hinode/EIS Ar XIV intensity and Ar XIV/Ca XIV ratio maps without and with SDO/HMI contours of \pm 500 (white/magenta for positive/ negative polarities) on 2017 September 7 at 08:01 UT. Bottom panel: Hinode/EIS Ar XIV 194.40 ^ and Ca XIV 193.87 A intensity maps, Ar XIV/Ca XIV ratio maps without and with SDO/HMI contours on 2017 September 7 at 21:35 UT. $\left(\mathrm{P}_{0}\right.$ is located at $X=692^{\prime \prime}, Y=-232^{\prime \prime}$ at $08: 01 \mathrm{UT}$ and at $X=766^{\prime \prime}, Y=-222^{\prime \prime}$ at 21:35 UT.)

had returned to FIP effect composition in the Ar XIV/Ca XIV ratio maps. Approximately $13.5 \mathrm{hr}$ later, a C5.4 flare triggered a series of Hinode/EIS observations from 21:35 to 22:19 UT. Figure 5, bottom panel, shows a representative sample at 21:35 UT. I-FIP effect plasma was no longer visible within the active region. Only remnant patches of photospheric composition remained in the vicinity of $\mathrm{N}_{4 u}$. Coronal composition was observed everywhere else including at $\mathrm{p}_{2}$ where patches of I-FIP effect plasma were present on the previous day.

\section{Discussion and Interpretation}

In this study, we have analyzed the evolution of plasma composition in AR 12673 on 2017 September 5-7. Hinode/ EIS detected highly localized patches of I-FIP effect plasma embedded within the characteristic FIP effect plasma of the active region core (e.g., Del Zanna \& Mason 2014; Baker et al. 2015). I-FIP effect plasma was present $\sim 8 \mathrm{hr}$ before the $\mathrm{X} 9.3$ class flare at 12:02 UT on September 6 and also during the long decay phase extending to $\sim 20: 00$ UT. The plasma composition 

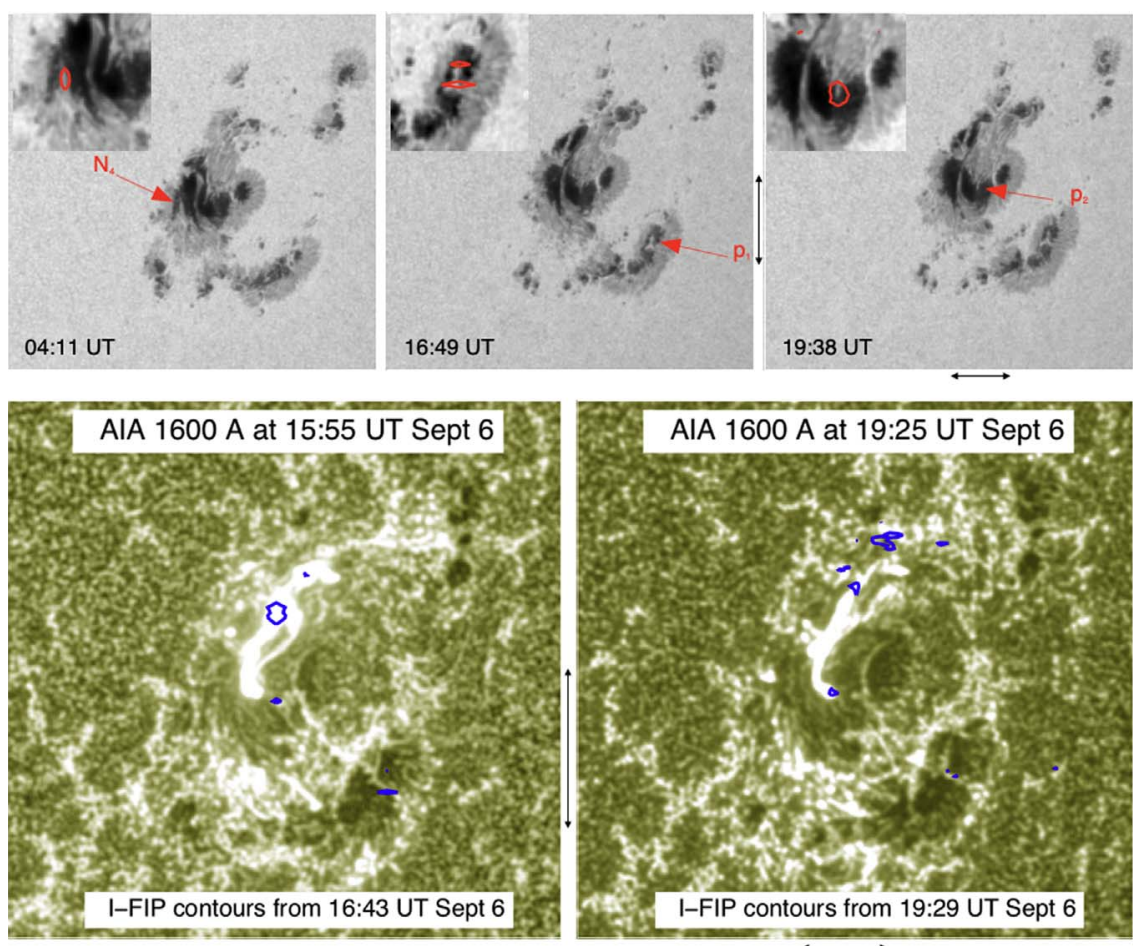

Figure 6. Top panels (left to right): SDO/HMI continuum images at 04:11, 16:49, and 19:38 UT on 2017 September 6. Red arrows indicate locations of strong light bridges at $\mathrm{N}_{4}, \mathrm{p}_{1}$, and $\mathrm{p}_{2}$ regions defined in Figure 2. Insets are zoomed images of the light bridges overplotted with red contours of the I-FIP effect $=1.3$. The I-FIP effect plasma is precisely at the locations of the strong light bridges. Bottom panels: SDO/AIA $1600 \AA$ images overplotted with blue I-FIP effect plasma contours of $\geqslant 1.3$. Contours have been derotated from the times of the Hinode/EIS rasters. $\left(\mathrm{P}_{0}\right.$ is located at $X=610^{\prime \prime}, Y=-241^{\prime \prime}$ at $19: 38 \mathrm{UT}$ and at $X=608^{\prime \prime}, Y=-241^{\prime \prime}$ at 19:25 UT.)

of AR 12673 had returned to FIP effect when Hinode/EIS observed it $\sim 13 \mathrm{hr}$ later.

\subsection{I-FIP Effect Plasma Observed at the Locations of Subphotospheric Reconnection}

A notable feature of the evolution of the highly complex magnetic field of AR 12673 is the coalescence of same polarity magnetic field strands in a number of locations throughout the active region. From the time of its emergence on September 3, the positive polarity strands of the $\mathrm{E}-\mathrm{W}$ oriented arcade system merged together $\mathrm{E}$ of $\mathrm{P}_{0}$ until a coherent umbra was evident at $\mathrm{p}_{2}$ in the continuum images early on September 4 (see the animation in Figure 1). A train of positive umbrae approaching from the $\mathrm{N}$ continued to coalesce with the already coherent $\mathrm{p}_{2}$ umbra for the next $\sim 2$ days before the structure began to fragment on September 7.

The main $\mathrm{N}-\mathrm{S}$ oriented flux system emerged after the arcade system later on September 3 when the opposite polarities rapidly separated, $\mathrm{N}_{4}$ moving in a northward direction and $\mathrm{P}_{4}$ progressing to the $\mathrm{S}-\mathrm{SW}$. Right from the start, there was a continuous influx of new strands of negative polarity coalescing at $\mathrm{N}_{4}$ for the same time period as with $\mathrm{p}_{2}$. Cotemporal emergence of $\mathrm{P}_{4}$ with $\mathrm{N}_{4}$ forced the positive field in the vicinity of $\mathrm{p}_{1}$ to amalgamate into the sunspot in the SW of the active region. The process of sunspot coalescence driven by strong flux emergence of same polarity field was repeated in $\mathrm{n}_{2}$ when $\mathrm{N}_{4 u}$ broke away from the main $\mathrm{N}_{4}$ umbra. It pushed into the negative field at $\mathrm{n}_{2}$ and then rotated around $\mathrm{P}_{3}$.

I-FIP effect plasma was observed within the coalescing umbrae at $\mathrm{p}_{2}, \mathrm{~N}_{4}, \mathrm{P}_{4}-\mathrm{p}_{1}$, and $\mathrm{N}_{4 u}-\mathrm{n}_{2}$. The patches can be located more precisely within the umbrae where strong LBs formed during the merging and coalescence of the sunspots. So-called strong LBs appear between magnetic field strands of the same polarity during the early stages of the active region's development rather than during sunspot decay which is more commonly associated with faint LBs (e.g., Felipe et al. 2016, and references therein). Red arrows indicate the locations of strong LBs within $\mathrm{N}_{4}, \mathrm{p}_{2}$, and $\mathrm{p}_{1}$ in the HMI continuum images from September 6 in Figure 6 (top panels). Contours of I-FIP effect plasma are overplotted on zoomed images of the LBs. The I-FIP effect plasma is precisely located at the LBs within the coalescing umbrae of the respective regions.

Once major flux emergence paused in the core of the active region late on September 6, the LBs were either no longer visible $\left(\mathrm{N}_{4}\right)$ or they became part of the fragmentation process in the sunspot during its decay phase $\left(\mathrm{p}_{2}\right)$. On the southern edge of the active region, the LB at $\mathrm{p}_{1}$ remained prominent for at least another day as flux emergence was still ongoing, driving $\mathrm{P}_{4}$ into $\mathrm{p}_{1}$ and forcing coalescence of the sunspot. The evolution of the LBs can be viewed in the HMI continuum images shown in the animated version of Figure 1.

Coalescing sunspot umbrae are preferential sites for subchromospheric reconnection to take place, especially during the early development of highly complex active regions. Major flux emergence episodes push flux tubes of the same polarity against each others. From their different subphotospheric evolution, these flux tubes are expected to typically have a finite angle between them. Then, component magnetic reconnection is expected to be present between the coalescing flux tubes (see Figure 7(a)).

There is a plausible alternative scenario of internal reconnection within an individual flux tube in the case of $\mathrm{N}_{4 u}$. The flux tube may have been deformed as it rotated around $\mathrm{P}_{3}$, creating 
(a) Reconnection

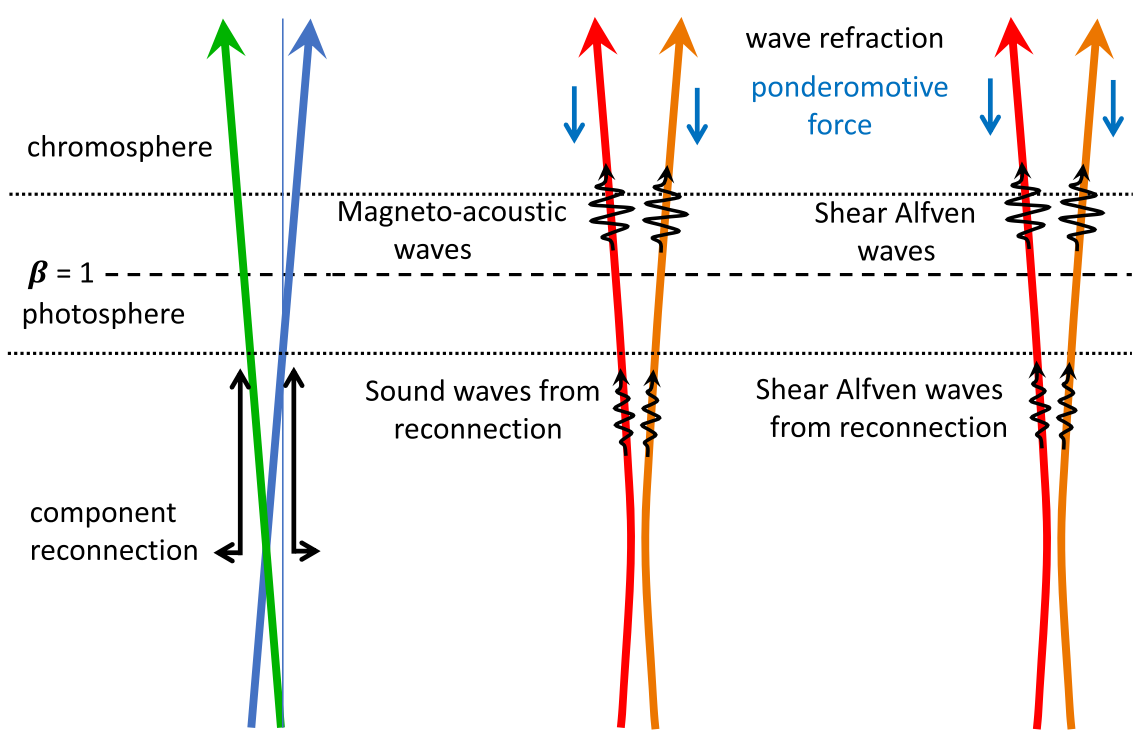

Figure 7. (a) Two flux systems with a nonzero component of antiparallel field between them are pushed together and reconnect below the photosphere. In the case of the general scenario shown in (b), reconnection generates sound waves that mode convert into magnetoacoustic waves at the $\beta=1$ layer. These waves are refracted/ reflected in the chromosphere, leading to a downward oriented ponderomotive force which depletes the low-FIP ions from the chromosphere. The general scenario is consistent with Baker et al. (2019). In the specific light bridge scenario (c) proposed in Section 4.4, subphotospheric reconnection launches incompressible (shear) Alfvén waves along the magnetic field lines at the edges of the light bridge. No mode conversion takes place but the rest of the scenario in (b) remains.

Table 3

Flare Class, Flare Start, Peak, and End Times from 2017 September 6 with the Locations of Flare Ribbons Corresponding to Figures 1 and 2

\begin{tabular}{lcc}
\hline \hline Flare Class & $\begin{array}{c}\text { Start/Peak/End } \\
\text { Times (UT) }\end{array}$ & $\begin{array}{c}\text { Flare } \\
\text { Ribbons }\end{array}$ \\
\hline C2.7 & $07: 29 / 07: 34 / 07: 48$ & $\mathrm{p}_{2}, \mathrm{~N}_{3}-\mathrm{N}_{4}$ \\
X2.2 & $08: 57 / 09: 10 />11: 53$ & $\mathrm{p}_{2}, \mathrm{~N}_{3}-\mathrm{N}_{4}$ \\
X9.3 & $11: 53 / 12: 02 />15: 51^{\mathrm{a}}$ & $\mathrm{p}_{2}-\mathrm{P}_{3}-\mathrm{P}_{4}, \mathrm{~N}_{4 u}-\mathrm{N}_{4}$ \\
M2.5 & $15: 51 / 15: 56 />19: 21$ & $\mathrm{p}_{2}-\mathrm{P}_{3}, \mathrm{~N}_{3}-\mathrm{N}_{4 u}-\mathrm{N}_{4 f}$ \\
M1.4 & $19: 21 / 19: 30 />23: 33$ & $\mathrm{p}_{2}-\mathrm{P}_{3}, \mathrm{~N}_{3}-\mathrm{N}_{4 u}-\mathrm{N}_{4 f}$ \\
M1.2 & $23: 33 / 23: 39 / 23: 44$ & $\mathrm{p}_{2}, \mathrm{~N}_{4 u}-\mathrm{N}_{4 f}$ \\
\hline
\end{tabular}

Notes. Flare details are from LMSAL SolarSoft latest events: http://www. lmsal.com/solarsoft/latest_events_archive/.

${ }^{a}$ The X9.3 flare loops are still present well after this time so that the decay phase of the X9.3 flare is mixed with the following flares. This is also the case for other $\mathrm{M}$ and $\mathrm{X}$ flares.

magnetic shear, beginning at around the time of the X2.2 flare and continuing during the early decay phase of the X9.3 flare. This evolution is shown from early on September 6 in the animation linked to Figure 1. Significant deformation of the $\mathrm{N}_{4 u}$ flux tube may generate internal current sheets where subphotospheric reconnection could take place. Either scenario or a combination of them is plausible at $\mathrm{N}_{4 u}$, however internal reconnection does not appear to be a likely cause of reconnection at $\mathrm{p}_{2}, \mathrm{~N}_{4}$, and $\mathrm{p}_{1}$ which showed little evidence of magnetic shear in their formation.

\subsection{Revealing I-FIP Effect Plasma with Flare Energy Input}

Successive episodes of major flux emergence and its interaction with a preexisting, well-anchored sunspot resulted in the formation of the highly complex magnetic structure of AR 12673. Continual interaction gave rise to significant flare productivity (e.g., Yang et al. 2017; Romano et al. 2018, 2019; Verma 2018; Toriumi \&
Wang 2019), indicating the occurrence of magnetic reconnection in the solar corona.

Evidence of significant localized heating generated by flaring is provided in Table 3 which lists the details for all flares $>$ C1.0 on September 6: flare class, timings (start/peak/end), and locations of flare ribbons. The locations of the flare ribbons were identified either explicitly as ribbons in the SDO/AIA (Lemen et al. 2012) $1600 \AA$ or in AIA $193 \AA$ images (not shown) as postflare loops, the coronal counterpart of the ribbons. Major flaring occurred in the vicinity of the U-loop configuration introduced in Section 2 (Yang et al. 2017; Yan et al. 2018).

During the rise phase of the $\mathrm{X} 9.3$ flare, two ribbons began to develop on either side of the C-shaped PIL within the active region core involving mainly $\mathrm{P}_{3}-\mathrm{p}_{2}-\mathrm{P}_{4}$ and $\mathrm{N}_{4 u}-\mathrm{N}_{4}$. The ribbons then extended into $\mathrm{N}_{4 f}$ and $\mathrm{N}_{3}$ soon after the eruption of the magnetic flux rope/CME coinciding with the flare peak at 12:02 UT (Mitra et al. 2018). The overlying postflare loop arcade rooted in the two flare ribbons remained visible in the SDO/AIA coronal passbands throughout the extended decay phase of the flare until the early hours of September 7.

Ribbons associated with the M2.5 and M1.4 flares (peaking at 15:56 and 19:30 UT, respectively) also appeared along the $\mathrm{PIL}$, tracing parts of the $\mathrm{X} 9.3$ ribbon "tracks" at $\mathrm{p}_{2}-\mathrm{P}_{3}$ and $\mathrm{N}_{3}-\mathrm{N}_{4 u}-\mathrm{N}_{4 f}$. The ribbons are clearly evident in SDO/AIA $1600 \AA$ images at 15:55 and 19:25 UT in the bottom panel of Figure 6. Blue contours of I-FIP effect plasma (ratio $\geqslant 1.3$ ) are overplotted on the images and have been derotated from the times of the Hinode/EIS raster times given in the figure. Contours located at $\mathrm{p}_{2}$ and $\mathrm{N}_{4 u}$ lie directly along the ribbons of both flares as does the $n_{2}$ contour associated with the M2.5 flare. There are no obvious flare ribbons extending to $\mathrm{P}_{4}$ in the AIA $1600 \AA$ image at 15:55 UT, however, the I-FIP effect contours correspond to the footpoints of the postflare arcade at 16:43 UT (not shown). 


\subsection{Comparison of ARs 11429 and 12673}

The Hinode/EIS observations of plasma composition in AR 12673 are globally comparable to those of AR 11429 (Baker et al. 2019). Distinct patches of I-FIP effect plasma were located at coalescing sunspot umbrae during flaring activity. The patches were observed when and where flare ribbons crossed the umbrae.

In the case of AR 11429, two compact patches of I-FIP effect plasma appeared and disappeared within one hour during the decay phase of a single, isolated M-class flare. Though the scenarios are very similar, the scales are different for AR 12673 in that four patches were present for timescales of hours not minutes over the merging umbrae of four instead of two sunspots. Furthermore, soft X-ray emission was significantly elevated for hours leading up to the first X-class flare and continued at very high levels for over $16 \mathrm{hr}$ after the X9.3 flare peak (Figure 2), compared to less than two hours during the M-class flare in AR 11429. The two M-class flares that occurred during the extended decay phase of the larger of the two X flares added additional heating sources. This does not necessarily mean that an increase in flaring activity directly produces more I-FIP effect plasma, since more flaring would only allow us to observe more I-FIP effect plasma regions via chromospheric evaporation if they are present.

However, more globally, an increase of magnetic complexity can induce more reconnection below the fractionation region (in the chromosphere) and in the corona, implying both the creation of more I-FIP effect plasma and more flares. Then, we expect a generic correlation between the complexity and emergence rate of the magnetic field, the amount of I-FIP effect plasma, and the flaring activity level.

Another common feature to both active regions is the occurrence of patches of I-FIP effect plasma at LBs within the coalescing umbrae. After noticing the precise location of the patches at the strong LBs during the formation stages of the same polarity sunspot umbrae, we reexamined the continuum movie of AR 11429 and indeed, patches of I-FIP effect plasma were also found under similar circumstances at strong LBs.

\subsection{Why is I-FIP Effect Plasma Observed at Light Bridges?}

Baker et al. (2019) proposed that transient patches of I-FIP effect plasma observed in AR 11429 were created by increased fast-mode wave flux that was generated by subchromospheric/ photospheric reconnection of coalescing umbrae (Figure 7(b)). Fast-mode waves coming from below the fractionation region of the chromosphere and undergoing a total internal reflection mean that the ponderomotive force is directed downward so that low-FIP elements are depleted from the chromospheric plasma (Laming 2015). Sunspot umbrae are preferential locations to observe I-FIP effect plasma. They are where the magnetic field is strongest, therefore the plasma $\beta=1$ layer is lower in the photosphere (Avrett et al. 2015) so that the fastmode wave flux from below is enhanced. The vertical field of the umbrae is also where the downward-directed ponderomotive acceleration is likely to be the strongest as the plasma upflow must be along the magnetic field, making the fieldaligned ponderomotive force most relevant.

EIS observed I-FIP effect plasma patches in four regions where subchromospheric or even subphotospheric reconnection was likely to be an ongoing process. In all cases, significant flux emergence was the main driver of sunspot coalescence.
This is consistent with the findings of Baker et al. (2019). The classical scenario is forced magnetic reconnection between two independent flux tubes where there is some angle between the same polarity strands that are pushed together by convective motions (Figure 7(a)), leading to the formation of MHD waves in the process. AR 12673 is an extreme case of the forced reconnection scenario due to the amount and complexity of the emerging magnetic field. There is essentially a rescaling due to energy input. Finally, I-FIP effect plasma was not observed within the active region after major flux emergence of the main flux system ceased.

Previously, we have argued in Baker et al. (2019) that fastmode waves cause the I-FIP effect, mainly because of the ease, relative to Alfvén waves, of achieving the required degree of reflection to produce I-FIP rather than FIP effect fractionation. Fast mode waves (sound waves) produced below the $\beta=1$ layer by reconnection mode convert where $\beta=1$ to continue as fast modes (now magnetoacoustic waves) in the $\beta<1$ chromosphere, where they refract/reflect exerting a downward ponderomotive force on the ions (Figure 7(b)). The mode conversion is essential, and increases with increasing angle between the magnetic field direction and the wavevector. While this might produce some selectivity in where I-FIP can be produced, upward-propagating sound waves are less likely to mode convert and produce I-FIP in the vertical magnetic field, and it is still less obvious why I-FIP should be restricted to the LBs, as observed in AR 11429 and AR 12673.

We propose the following slightly more refined scenario, which consists of two interlinked parts: the first ingredient is the geometry of the subphotospheric reconnection that creates the train of waves responsible for the I-FIP effect fractionation; the second one is the nature of waves such that they remain associated with a specific bundle of field lines, emerging at a very specific location, and only there.

LBs are very particular areas within an active region where pockets of relatively unmagnetized plasma are trapped within two merging flux systems of the same strong polarity, forming a sunspot. The subphotospheric forces that merge the two flux systems may eventually be able to squeeze out the unmagnetized plasma and lead to a structure that looks like a singlepolarity spot in white light images and line-of-sight magnetograms. Therefore, LBs are the photospheric trace of the merging plane between two flux systems that, generally, extend down in the convection zone, at least deeper than the vertical extension of the unmagnetized plasma volume that supports the LB. At the subphotospheric location where the two flux systems are pushed together by the high- $\beta$ plasma, it is conceivable that component field reconnection between nearly parallel flux bundles of the same sign occurs (Figure 7(a)). This is the main assumption of this scenario. If this is true, then the numerical simulations by Kigure et al. (2010) provide crucial information, namely that in a high- $\beta$ plasma environment, the reconnection between nearly parallel flux systems would mostly generate incompressible (shear) Alfvén waves. Applying this result in our hypothetical scenario (Figure 7(c)), the subphotospheric reconnection process would then launch incompressible Alfvén waves along the field lines of the flanks of the reconnecting flux systems that surround the LB. Since Alfvén waves must propagate along field lines, as they travel upward, these waves would then eventually emerge at the sides of the LB, irrespective of how deep the LB extends below the photosphere. Furthermore, the magnetic field right above a LB 
was observed recently by Felipe et al. (2016) using high resolution measurements from the GREGOR telescope (Schmidt et al. 2012). The authors conclude that the field at the side of the LB stretches up along its two sides and converges above the LB, forming a cusp shape across it, with the mainly horizontal LB field being confined below the cusp. Following the cusp lines, the shear Alfvén waves of subphotospheric origin would reach the chromosphere, where they could provide the energy, and the correct direction of travel, for the ponderomotive I-FIP effect fractionation in a very localized volume, namely right above the LB. Hence, if the above scenario is correct, the observations of I-FIP effect fractionated plasma should be located between the merging sunspot's fragments, i.e., at the LB, which is indeed what is observed.

The above scenario is highly speculative. Getting sufficient reflection of Alfvén waves to cause I-FIP rather than FIP effect fractionation probably requires more wave interaction physics in the chromospheric model, as Alfvén waves likely reflect off sound waves, shocks, or other density inhomogeneities. This can be modeled analytically, but will require both numerical testing and observational confirmation. The numerical testing is needed to confirm that upwards traveling shear Alfvén waves from below the photosphere can reflect efficiently and drive the I-FIP effect fractionation. The observational verification entails a multiline spectropolarimetric study of LBs to verify the presence of such waves at the sides of the LB. On the other hand, such a scenario assumes subphotospheric reconnection between the merging flux systems that drives the formation of an observed LB and therefore determines the observed localization of I-FIP effect plasma on the basis of the properties of reconnection between nearly parallel flux systems. As mentioned above, such a localization at LBs is more difficult to explain with compressible waves which can travel across field lines.

\section{Conclusions}

I-FIP effect plasma has been detected in only eight active regions (Doschek et al. 2015; Doschek \& Warren 2016, 2017), but it is not yet clear how widespread the phenomenon is. Observational constraints are likely to play a role in limiting the number of I-FIP effect plasma detections. It requires the right composition lines in the employed study, the correct target selection, and pointing at the specific flaring locations within the target active region during the time of flaring activity.

A significant fraction of the I-FIP effect plasma observations were obtained while Hinode/EIS was operating in its flare trigger mode. This is an observing strategy to catch flares, and the response study is initiated only at or near the flare peak time. It remains to be seen whether a different observing strategy, dedicated to observing I-FIP effect events, would be more successful at capturing the evolution of plasma composition before and during the flare rise phase.

Notwithstanding these limitations, observations of AR 11429 and AR 12673 show that they share common characteristics in their magnetic configuration which distinguish them as potential candidates for the detection of I-FIP effect plasma. Both active regions were large (>500 MSH) and magnetically complex $(\beta \gamma \delta)$ with ongoing major flux emergence of sheared/twisted field which violently interacted with the preexisting field.
Our interpretation of the Hinode/EIS observations of I-FIP effect plasma in AR 12673 is consistent with the ponderomotive fractionation model. Ongoing subchromospheric or subphotospheric reconnection at multiple sunspot umbrae is likely to increase the wave flux from below the chromosphere, thereby providing favorable circumstances for the accumulation of I-FIP effect plasma in more locations and on longer timescales than for AR 11429.

Flaring and evaporation of chromospheric material are essential parts of this scenario. Along flare ribbons at the footpoints of newly formed loops, which rarely traverse coalescing umbrae, the expected chromospheric composition is close to photospheric. The Sun-as-a-star (Warren 2014) and stellar (Audard et al. 2003; García-Alvarez et al. 2009; Testa et al. 2015) observations during flares are consistent with this, since they indicate that the overall elemental composition is getting close to the photospheric value. The presence of bright I-FIP patches shifts the overall composition of FIP-effect dominated coronae even more toward photospheric or indeed to I-FIP effect values (e.g., Katsuda et al. 2020).

Do we need "monster" active regions to produce I-FIP effect coronal composition? So far the evidence is pointing that way, but the picture is clouded by observational constraints. What is likely to be a requirement is a strong wave flux from below the chromospheric fractionation region. There is a considerable fraction, $\sim 30 \%$, of the total released energy of Alfvén waves generated in high plasma- $\beta$ reconnection events (Kigure et al. 2010), so that I-FIP effect is expected to occur more often than what has been observed so far if subphotospheric reconnection occurs frequently between the multiple thin flux tubes present in emerging active regions. Based on the observations of AR 12673, we propose a scenario where shear Alfvén waves originating below the photosphere and traveling upward can localize the I-FIP effect plasma at strong light bridges at coalescing umbrae.

Active region complexity is highly correlated with activity (see e.g., Toriumi \& Wang 2019, and references therein) so there is a temptation to associate the presence of localized regions of I-FIP effect plasma in so-called monster active regions on the Sun with more active $M$ dwarf stars containing very large/strong starspots (e.g., Berdyugina 2005; Reiners 2012) and whose coronae are dominated by I-FIP effect plasma. It is expected to be a matter of the scale of waves generated by magnetic reconnection in subfractionation layers of stars. X-ray flux is a function of overall heating in a star's atmosphere (e.g., Wood et al. 2018) which in turn governs the elements that are ionized on that star. The presence of a strong and highly complex magnetic field provides additional heating and MHD waves that determine the direction and strength of the ponderomotive force and therefore the degree and direction of plasma fractionation, whether I-FIP or FIP effect, on stars of spectral types F-M.

The filling factor of spot umbrae on the photosphere of a star is likely to be a significant factor in determining the extent to which I-FIP effect plasma fills the star's corona. On the Sun, strong magnetic fields are found in the small localized areas of sunspots covering a small fraction of the surface $(<0.5 \%$, Hathaway 2015), hence we may observe very small patches of I-FIP effect plasma. On the more active $\mathrm{M}$ dwarfs, starspots can cover up to two orders of magnitude more of a star's 
photosphere (e.g., Jackson \& Jeffries 2013; Tregloan-Reed \& Unda-Sanzana 2019), which may explain why their coronae are dominated by I-FIP effect plasma.

The recognition that stellar coronal composition has a dependence on magnetic activity (Audard et al. 2003; GarcíaAlvarez et al. 2009; Testa et al. 2015) is in line with our results of resolved I-FIP generation on the Sun in a FIP-effect dominated low-activity star. The I-FIP patches are closely related to strong magnetic field concentrations and made observable in the corona by flaring.

We thank the referee for their helpful suggestions to improve the clarity of the manuscript. The authors are very grateful to Konkoly Observatory, Budapest, Hungary, for hosting two workshops on Elemental Composition in Solar and Stellar Atmospheres (IFIPWS-1, 2017 February 13-15 and IFIPWS-2, 2018 February 27-March 1) and acknowledge the financial support from the Hungarian Academy of Sciences under grant NKSZ 2018_2. The workshops have fostered collaboration by exploiting synergies in solar and stellar magnetic activity studies and exchanging experience and knowledge in both research fields. Hinode is a Japanese mission developed and launched by ISAS/JAXA, collaborating with NAOJ as a domestic partner, and NASA and STFC (UK) as international partners. Scientific operation of Hinode is performed by the Hinode science team organized at ISAS/JAXA. This team mainly consists of scientists from institutes in the partner countries. Support for the postlaunch operation is provided by JAXA and NAOJ (Japan), STFC (UK), NASA, ESA, and NSC (Norway). SDO data were obtained courtesy of NASA/SDO and the AIA and HMI science teams. D.B. is funded under STFC consolidated grant No. ST/S000240/1 and L.v.D.G. is partially funded under the same grant. The work of D.H.B. was performed under contract to the Naval Research Laboratory and was funded by the NASA Hinode program. G.V. acknowledges the support from the European Union's Horizon 2020 research and innovation programme under grant agreement No. 824135 and of the STFC grant No. ST/T000317/1 J. M.L. was supported by the NASA HGI (80HQTR19T0029), NASA HSR (NNH16AC391), and LARS (NNH17AE601) programs, the Chandra GO program, and by Basic Research Funds of the Chief of Naval Research. DML is grateful to the Science Technology and Facilities Council for the award of an Ernest Rutherford Fellowship (ST/R003246/1).

\section{ORCID iDs}

Deborah Baker (iD https://orcid.org/0000-0002-0665-2355 Lidia van Driel-Gesztelyi (i) https://orcid.org/0000-00022943-5978

David H. Brooks (iD https://orcid.org/0000-0002-2189-9313 Pascal Démoulin (iD https://orcid.org/0000-0001-8215-6532 Gherardo Valori (iD https://orcid.org/0000-0001-7809-0067 David M. Long (iD https://orcid.org/0000-0003-3137-0277 J. Martin Laming (iD https://orcid.org/0000-0002-3362-7040 Andy S. H. To (i) https://orcid.org/0000-0003-0774-9084 Alexander W. James (iD https://orcid.org/0000-0001-7927-9291

\section{References}

Anfinogentov, S. A., Stupishin, A. G., Mysh'yakov, I. I., \& Fleishman, G. D. 2019, ApJL, 880, L29

Audard, M., Güdel, M., Sres, A., Raassen, A. J. J., \& Mewe, R. 2003, A\&A, 398,1137
Avrett, E., Tian, H., Landi, E., Curdt, W., \& Wülser, J. P. 2015, ApJ, 811, 87 Baker, D., Brooks, D. H., Démoulin, P., et al. 2015, ApJ, 802, 104

Baker, D., Brooks, D. H., van Driel-Gesztelyi, L., et al. 2018, ApJ, 856, 71 Baker, D., van Driel-Gesztelyi, L., Brooks, D. H., et al. 2019, ApJ, 875, 35 Berdyugina, S. V. 2005, LRSP, 2, 8

Bobra, M. G., Sun, X., Hoeksema, J. T., et al. 2014, SoPh, 289, 3549

Brooks, D. H. 2018, ApJ, 863, 140

Brooks, D. H., Baker, D., van Driel-Gesztelyi, L., \& Warren, H. P. 2017, NatCo, 8, 183

Brooks, D. H., Ugarte-Urra, I., \& Warren, H. P. 2015, NatCo, 6, 5947

Brown, C. M., Feldman, U., Seely, J. F., Korendyke, C. M., \& Hara, H. 2008, ApJS, 176, 511

Caffau, E., Ludwig, H.-G., Steffen, M., Freytag, B., \& Bonifacio, P. 2011, SoPh, 268, 255

Culhane, J. L., Harra, L. K., James, A. M., et al. 2007, SoPh, 243, 19

Del Zanna, G., Dere, K. P., Young, P. R., Landi, E., \& Mason, H. E. 2015, A\&A, 582, A56

Del Zanna, G., \& Mason, H. E. 2014, A\&A, 565, A14

Dere, K. P., Landi, E., Mason, H. E., Monsignori Fossi, B. C., \& Young, P. R. 1997, A\&AS, 125, 149

Doschek, G. A., Mariska, J. T., Warren, H. P., et al. 2007, PASJ, 59, S707

Doschek, G. A., \& Warren, H. P. 2016, ApJ, 825, 36

Doschek, G. A., \& Warren, H. P. 2017, ApJ, 844, 52

Doschek, G. A., Warren, H. P., \& Feldman, U. 2015, ApJL, 808, L7

Fan, Y., Abbett, W. P., \& Fisher, G. H. 2003, ApJ, 582, 1206

Feldman, U. 1992, PhyS, 46, 202

Feldman, U., Warren, H. P., Brown, C. M., \& Doschek, G. A. 2009, ApJ, 695,36

Feldman, U., \& Widing, K. G. 1990, ApJ, 363, 292

Felipe, T., Collados, M., Khomenko, E., et al. 2016, A\&A, 596, A59

Freeland, S. L., \& Handy, B. N. 1998, SoPh, 182, 497

García-Alvarez, D., Drake, J. J., \& Testa, P. 2009, in AIP Conf. Ser. 1094, XV Cambridge Workshop on Cool Stars, Stellar Systems, and the Sun, ed. E. Stempels (Melville, NY: AIP), 796

Graham, D. R., Fletcher, L., \& Hannah, I. G. 2011, A\&A, 532, A27

Hathaway, D. H. 2015, LRSP, 12, 4

Jackson, R. J., \& Jeffries, R. D. 2013, MNRAS, 431, 1883

Katsuda, S., Ohno, M., Mori, K., et al. 2020, ApJ, 891, 126

Kigure, H., Takahashi, K., Shibata, K., Yokoyama, T., \& Nozawa, S. 2010, PASJ, 62, 993

Kosugi, T., Matsuzaki, K., Sakao, T., et al. 2007, SoPh, 243, 3

Laming, J. M. 2015, LRSP, 12, 2

Laming, J. M. 2017, ApJ, 844, 153

Laming, J. M., Drake, J. J., \& Widing, K. G. 1995, ApJ, 443, 416

Laming, J. M., \& Hwang, U. 2009, ApJL, 707, L60

Lemen, J. R., Title, A. M., Akin, D. J., et al. 2012, SoPh, 275, 17

Lodders, K. 2008, ApJ, 674, 607

Mitra, P. K., Joshi, B., Prasad, A., Veronig, A. M., \& Bhattacharyya, R. 2018, ApJ, 869, 69

Nordon, R., \& Behar, E. 2008, A\&A, 482, 639

Redmon, R. J., Seaton, D. B., Steenburgh, R., He, J., \& Rodriguez, J. V. 2018, SpWea, 16, 1190

Reiners, A. 2012, LRSP, 9, 1

Romano, P., Elmhamdi, A., Falco, M., et al. 2018, ApJL, 852, L10

Romano, P., Elmhamdi, A., \& Kordi, A. S. 2019, SoPh, 294, 4

Scherrer, P. H., Schou, J., Bush, R. I., et al. 2012, SoPh, 275, 207

Schmidt, W., von der Lühe, O., Volkmer, R., et al. 2012, AN, 333, 796

Simões, P. J. A., Graham, D. R., \& Fletcher, L. 2015, A\&A, 577, A68

Sun, X., \& Norton, A. A. 2017, RNAAS, 1, 24

Sylwester, J., Lemen, J. R., \& Mewe, R. 1984, Natur, 310, 665

Testa, P., Saar, S. H., \& Drake, J. J. 2015, RSPTA, 373, 20140259

Toriumi, S., Hayashi, K., \& Yokoyama, T. 2014, ApJ, 794, 19

Toriumi, S., \& Wang, H. 2019, LRSP, 16, 3

Tregloan-Reed, J., \& Unda-Sanzana, E. 2019, A\&A, 630, A114

Tripathi, D., Mason, H. E., Young, P. R., \& Del Zanna, G. 2008, A\&A, 481, L53

van Driel-Gesztelyi, L., Malherbe, J. M., \& Démoulin, P. 2000, A\&A, 364, 845 Verma, M. 2018, A\&A, 612, A101

Wang, H., Yurchyshyn, V., Liu, C., et al. 2018, RNAAS, 2, 8

Warren, H. P. 2014, ApJL, 786, L2

Watanabe, T., Hara, H., Sterling, A. C., \& Harra, L. K. 2010, ApJ, 719, 213

Wood, B. E., Laming, J. M., Warren, H. P., \& Poppenhaeger, K. 2018, ApJ, 862,66

Wood, B. E., \& Linsky, J. L. 2010, ApJ, 717, 1279

Yan, X. L., Wang, J. C., Pan, G. M., et al. 2018, ApJ, 856, 79

Yang, S., Zhang, J., Zhu, X., \& Song, Q. 2017, ApJL, 849, L21 